\title{
(GIGA)byte
}

\section{Genomic Resources for the North American Water Vole (Microtus richardsoni) and the Montane Vole (Microtus montanus)}

\author{
Drew J. Duckett ${ }^{1, *}$, Jack Sullivan ${ }^{2}$, Stacy Pirro ${ }^{3}$ and Bryan C. Carstens ${ }^{1}$
}

1 Department of Evolution, Ecology, and Organismal Biology, The Ohio State University, 1315 Kinnear Rd., Columbus, OH 43212, USA

2 Department of Biological Sciences, University of Idaho, Box 443051, Moscow, ID 83844-3051, USA

3 Iridian Genomes, Inc., 6213 Swords Way, Bethesda, MD 20817, USA

\section{ABSTRACT}

Voles of the genus Microtus are important research organisms, yet genomic resources are lacking. Such resources would benefit future studies of immunology, phylogeography, cryptic diversity, and more. We sequenced and assembled nuclear genomes from two subspecies of water vole (Microtus richardsoni) and from the montane vole (Microtus montanus). The water vole genomes were sequenced with Illumina and 10× Chromium plus Illumina sequencing, resulting in assemblies with $\sim 1600,000$ and $\sim 30,000$ scaffolds, respectively. The montane vole was also assembled into $\sim 13,000$ scaffolds using Illumina sequencing. Mitochondrial genome assemblies were also performed for both species. Structural and functional annotation for the best water vole nuclear genome resulted in $~ 24,500$ annotated genes, with $83 \%$ of these having functional annotations. Assembly quality statistics for our nuclear assemblies fall within the range of genomes previously published in the genus Microtus, making the water vole and montane vole genomes useful additions to currently available genomic resources.

Subjects Genetics and Genomics, Animal Genetics, Evolutionary Biology, Functional Genomics

Submitted: Accepted:

22 January 2021 Published:

04 May 2021 06 May 2021

* Corresponding author. E-mail: duckettdj@gmail.com

Published by GigaScience Press.

Preprint submitted at https: //doi.org/10.1101/2021.04.04.438380

This is an Open Access article distributed under the terms of the Creative Commons Attribution License (http://creativecommons.org/ licenses/by/4.0/), which permits unrestricted reuse, distribution, and reproduction in any medium, provided the original work is properly cited.

Gigabyte, 2021, 1-13

\section{DATA DESCRIPTION}

\section{Background}

The genus Microtus comprises 62 species of voles, distributed throughout North America, Europe, and Asia [1]. Microtus is believed to have undergone rapid speciation and diversification, with all speciation events occurring within the past 4 million years [2, 3]. It has been suggested that some nominal species, such as M. pennsylvanicus, contain cryptic diversity [4]. Microtus is an important model system across multiple biological disciplines, including studies of adaptation (e.g., [5]), infectious disease (e.g., [6]), parental care (e.g., [7]), and population dynamics (reviewed in [8]).

Currently, assembled genomes for four Microtus species have been deposited in GenBank: two European species (M. agrestis and M. arvalis) and two North American species (M. ochrogaster; [9], and M. oeconomus). The present study provides resources for two additional species: M. richardsoni (NCBI:txid111840) and M. montanus (NCBI:txid88450).

The North American water vole (M. richardsoni) occupies a large, disjunct distribution in the Pacific Northwest of North America, with habitat in the Cascades Mountains and the 
Rocky Mountains, spanning from southern Canada into central Utah. Four subspecies are currently recognized: M. r. arvicoloides in the Cascades Mountains, $M$. r. richardsoni in the Canadian Rocky Mountains, M. r. macropus in the central Rocky Mountains and Wyoming, and M. r. myllodontus in Utah. M. richardsoni is adapted to a semiaquatic lifestyle, relying on alpine and subalpine streams for creating burrows and escaping predators [10]. Like other semiaquatic mammals (e.g., otters), adaptations to this lifestyle have probably been driven by natural selection [11-13]. Water voles are among the largest species of Microtus, and their frequent movement makes runways of trampled vegetation along streams [10, 14]. Unlike most other vole species, $M$. richardsoni does not appear to experience regular population boom and bust cycles, although population size in the species may correlate with levels of precipitation [15]. Despite being listed in the International Union for Conservation of Nature (IUCN) Red List [16] as being of 'Least Concern', the species is listed as 'critically imperiled' in the Wyoming Natural Diversity Database owing to its specific habitat requirements, which can be substantially degraded by livestock grazing [17].

The montane vole (M. montanus) is partially sympatric with $M$. richardsoni, and can be found throughout most of the water vole's range, with the exception of the Canadian Rockies. However, M. montanus can be found farther south and east, including areas of California, Nevada, Colorado, Arizona, and New Mexico [18]. The species has been divided into 15 subspecies, including M. m. canescens in the Cascades Mountains, M. m. nasus in the central Rocky Mountains, and M. m. amosus in northern Utah. Notably, M. montanus does not exhibit a break in its range in the Columbia Basin; probably because it is not restricted to riparian areas like $M$. richardsoni. The species is IUCN Red-Listed as being of 'Least Concern'. However, M. m. arizonicus is 'endangered' according to the New Mexico State Game Commission Regulation [18], and M. m. ricularis is 'of concern', owing to its small range and declining population size [19].

\section{Context}

The rapid radiation of Microtus voles has hindered systematic classification, leading to multiple taxonomic revisions and conflicting phylogenetic analyses [1, 20, 21]. Consequently, both species boundaries and relationships between species are difficult to infer.

Genomic resources within Microtus will help resolve these questions, and resources have steadily increased in recent years. Here, we present two nuclear and one mitochondrial genome assembly for $M$. richardsoni, as well as a structural and functional annotation for one of the $M$. richardsoni genomes. The subspecific classifications and disjunct range of the species means that $M$. richardsoni has been included in multiple phylogeography studies in the Pacific Northwest [22-24]. These studies were based solely on mitochondrial DNA, and the results of analyses that investigated species limits and demographic history were limited to inferences that can be derived from a single gene tree. These genomic resources will provide a rich source of data to address these knowledge gaps and aid in future studies of adaptation.

We also present single nuclear and mitochondrial genome assemblies for M. montanus. Genomic resources in M. montanus will provide a wealth of data to assess subspecies boundaries, quantify gene flow among subspecies, and aid in conservation efforts of threatened subspecies. Genome-level comparisons are also made between the new genome assemblies and other Microtus genome assemblies to examine differences in assembly quality and repeat content. 


\section{METHODS}

\section{Sequencing and nuclear genome assembly}

Frozen tissue from a single M. $r$. arvicoloides individual collected from the southern Cascades Mountain range (JMS_292; 44.016667N, -121.750000E; [22]) was sent to Hudson Alpha (Huntsville, AL, USA) for high molecular weight DNA extraction and 10× Chromium library preparation [25]. In the $10 \times$ method, each extracted DNA fragment receives a different barcode before the fragment is sheared for library preparation. After sequencing, these barcodes are used to connect sequencing reads for a more contiguous assembly. After sequencing with a single run on an Illumina HiSeqX, the resulting 150-bp paired-end reads were input into Supernova v. 2.2.1 for de novo genome assembly with -maxreads=all [26].

Additional tissue was obtained from a single M. $r$. macropus individual collected from the northern Rocky Mountains (JMG_88; 46.333333N, -114.633333E; [22]). DNA was extracted using a Qiagen DNeasy Blood and Tissue Kit, and the DNA was sent for library preparation and sequencing by Iridian Genomes, Inc (Bethesda, MD, USA). Then, 150-bp paired-end reads were sequenced on two runs of an Illumina HiSeqX. Genome assembly was performed using two different de Bruijn graph-based programs, SOAPdenovo v. 2.04 (SOAP, RRID:SCR_000689) and Discovar de novo v. 52488 (Discovar assembler, RRID:SCR_016755) [27, 28]. For SOAPdenovo, quality trimming was performed using fastQC v. 0.11.8 (FastQC, RRID:SCR_014583) and Trimmomatic v. 0.38 (Trimmomatic, RRID:SCR_011848) with settings ILLUMINACLIP: 2:30:10, LEADING:3, TRAILING:3, SLIDINGWINDOW:4:15, and MINLEN:36 [29, 30]. SOAPdenovo assemblies were performed with settings max_rd_len $=150$, avg_ins $=300$, reverse_seq $=0$, asm_flags $=3$, rd_len_cutoff $=150$, rank=1, pair_num_cutoff $=3$, and map_len $=32$. SOAPdenovo was run with $k$-mer values of 63 , 89, 95, and 101 based on analysis of optimal $k$-mer values in kmerGenie v. 1.7051 [31]. Raw reads were used as input for de novo genome assembly with Discovar, as recommended in the program documentation.

To provide the most contiguous assembly for $M$. richardsoni, a hybrid assembly was performed using the ARCS+LINKS pipeline [32, 33]. The ARCS+LINKS pipeline uses barcoding information from the $10 \times$ Chromium reads to scaffold the contigs from a separate genome assembly. Barcoded reads from M. r. arvicoloides were mapped to the M. $r$. macropus Discovar assembly with bwa mem v. 0.7.17 [34] before converting the mapped reads to BAM format and sorting with SAMTools v. 1.8 (SAMTOOLS, RRID:SCR_002105) [35]. ARCS v. 1.0.5 and LINKS v. 1.8.6 were then run with settings $-\mathrm{s} 98-\mathrm{c} 5-\mathrm{l} 0-\mathrm{z} 500-\mathrm{d} 0-\mathrm{r} 0.05$ -m 50-10000 -e 30000 and -d 4000 -k 20 -l 5 -t 2 -a 0.3 -o 0 -a 0.3 -z 500, respectively.

As part of a separate project, a single $M$. montanus individual from Utah (UMNH:Mamm:30891; 38.19381N, -111.5824E) was misidentified as M. richardsoni. DNA was extracted from the sample using a Qiagen DNeasy Blood and Tissue Kit before being sent to the University of California Davis Genome Center for library preparation and sequencing. Then, 150-bp paired-end sequences were collected with a single shared run on an Illumina NovaSeq. Species identity was confirmed using the Barcode of Life Database (BOLD [36]). Reads were checked and trimmed for quality with fastQC and Trimmomatic as above, before mapping reads to the mitochondrial cytochrome oxidase I (COI) sequence of M. r. macropus [37] using bwa mem. The resulting mapped reads were converted to BAM format, sorted, and indexed with SAMTools. PCR duplicates were identified and removed with Picard v. 2.3.0 (Picard, RRID:SCR_006525) [38]. Resulting reads were then piled with SAMTools mpileup using base and mapping quality scores of 30 , consensus sequences were 
generated with bcftools v. 1.3.1 (SAMtools/BCFtools, RRID:SCR_005227) [39], and consensus sequences were converted to fastq format using vcfutils with a minimum depth filter of 5 and maximum depth filter of 10,000 [35]. The resulting sequence was input into BOLD. Owing to low sequencing coverage, de novo genome assembly was not appropriate for $M$. montanus. To provide a preliminary genome sequence, a reference-guided genome assembly was performed with RaGOO v. 1.1 [40]. Raw reads were input into Discovar to generate an initial genome assembly. Misassembly correction was performed with RaGOO, using reads trimmed with the same settings as the M. $r$. macropus reads, and RaGOO was then used to scaffold the Discovar contigs onto the M. $r$. arvicoloides assembly; this is more closely related to M. montanus than the other available Microtus genome assemblies [3]. Since $M$. montanus has less than half the chromosomes of $M$. richardsoni ( $2 n=22-24$ in $M$. montanus versus 56 in $M$. richardsoni [41]), the possibility of structural errors in the $M$. montanus assembly was examined by calculating the percentage of reads that mapped back to the assembly using bwa mem and bamtools v. 2.2.2 [42].

The final assemblies were submitted to GenBank [43], where screening was performed to identify any contamination, and contaminated scaffolds were removed. All assemblies were evaluated with QUAST v. 5.0.1 (QUAST, RRID:SCR_001228) [44], bbmap v. 38.35 (BBmap, RRID:SCR_016965) [45], custom Python scripts [46], and BUSCO v. 5.0.0 (BUSCO, RRID:SCR_015008) using the Euarchontoglires reference set [47]. After comparing assembly statistics from the different assemblies of M. r. macropus, the Discovar assembly was selected as the best one because it had less fragmentation, higher N50 and lower L50 values, and a higher BUSCO score than the SOAPdenovo assemblies (Table 1). Genome sequencing of $M$. r. arvicoloides produced over 800 million reads and $47 \times$ genome sequencing coverage. The final genome assembly consisted of $\sim 32,000$ scaffolds, with an N50 of $2.3 \mathrm{Mb}$ (megabase pairs), $1.3 \%$ missing data (N), and a BUSCO score of $85.8 \%$. Supernova estimated the length of the genome assembled to be $\sim 2.4 \mathrm{~Gb}$ (gigabase pairs) and the total genome size to be $\sim 2.6$ Gb. M. r. macropus sequencing produced over 600 million reads and 35× coverage. Genome assembly with Discovar resulted in $\sim 1.6$ million scaffolds, with an N50 of $16 \mathrm{~Kb}$ (kilobase pairs), $0.06 \% \mathrm{~N}$, and a BUSCO score of $54.5 \%$. Given the many programs available to perform de novo genome assembly from short reads, another program might have produced a more contiguous M. r. macropus assembly, but previous studies have shown that Discovar performs well compared to other programs [48, 49]. The hybrid assembly produced with the ARCS+LINKS pipeline had $~ 1.6$ million scaffolds, an N50 of $38 \mathrm{~Kb}, 0.09 \%$ $\mathrm{N}$, and a BUSCO score of $59.8 \%$. Because the hybrid assembly was of poor quality, it was not used for further analyses, and the $M$. richardsoni subspecies assemblies were kept separate. High fragmentation of the hybrid assembly is probably associated with fragmentation of the Discovar input assembly. Published results with this hybrid pipeline often include a much higher sequencing coverage of the input contigs to produce a better starting point for the pipeline. Therefore, in the future, additional Illumina sequencing with M. r. macropus might substantially improve the hybrid assembly. To produce the preliminary $M$. montanus genome, 108 million reads (13× coverage) were used, resulting in 13,000 scaffolds, an N50 of $\sim 3.1 \mathrm{Mb}, 8.8 \% \mathrm{~N}$, and a BUSCO score of $82.6 \%$. Additionally, $89.3 \%$ of reads mapped back to the M. montanus assembly.

\section{Mitochondrial genomes}

The complete mitochondrial genomes of $M$. r. arvicoloides and $M$. montanus were assembled using the genomic sequencing reads. Mitochondrial genomes were assembled by 


\begin{tabular}{cccccc}
\hline \multicolumn{6}{l}{ Table 1. Comparison of genome assembly strategies for Microtus richardsoni macropus. } \\
\hline & Discovar & SOAPdenovo & SOAPdenovo & SOAPdenovo & SOAPdenovo \\
\hline k-mer $(n)$ & NA & 63 & 89 & 95 & 101 \\
Length (Gb) & 2.54 & 2.72 & 2.88 & 2.89 & 3.21 \\
Scaffolds ( $n$, millions) & 1.6 & 4.1 & 4.0 & 4.1 & 6.7 \\
Max Scaffold (Kb) & 264 & 186 & 146 & 174 & 139 \\
N50 (Kb) & 16.1 & 4.5 & 3.4 & 3.4 & 1.5 \\
L50 ( $n$, thousands) & 35.7 & 117 & 156 & 163 & 371 \\
BUSCO (\%) & 54.5 & 38.1 & 37.1 & 35.9 & 25.9 \\
N (\%) & 0.06 & 1.45 & 0.99 & 0.94 & 0.90 \\
GC content (\%) & 42.13 & 41.92 & 41.91 & 41.92 & 41.98 \\
\hline NA: not applicable. & & & & & \\
\hline
\end{tabular}

both mapping reads to a reference mitochondrial genome, and by using the reference-guided assembly program Novoplasty v. 4.1 [50]. For the mapping assembly, reads were mapped to the $M$. $r$. macropus mitochondrial genome using the same steps as in the $M$. montanus BOLD analysis. The mitochondrial assemblies were 16,285 bp and $16,268 \mathrm{bp}$ in length, with an average depth of coverage of $7886 \times$ and $6805 \times$ for $M . r$. arvicoloides and $M$. montanus, respectively. Reference-guided mitochondrial assemblies with Novoplasty used the M. r. macropus mitochondrial genome as the reference, along with settings Genome Range $=12,000-22,000, K$-mer $=33$, Read Length $=150$, and Insert size $=400$. Because the $M$. $r$. arvicoloides dataset contained many reads, $25 \%$ of reads were subsampled for assembly, as suggested in the program documentation. The assemblies for M. r. arvicoloides and M. montanus were 16,298 bp and 16,319 bp in length, with average depths of coverage of $5131 \times$ and $14,713 \times$, respectively.

To compare mitochondrial assemblies between methods, the assemblies were aligned using the MUSCLE plugin in Geneious v. R9 (Geneious, RRID:SCR_010519) with eight iterations and an open gap score of -1 [51, 52]. This comparison showed that the Novoplasty assemblies contained multiple insertions compared with the mapped assemblies and the reference mitochondrial genome. These insertions were up to 13-bp long in multiple genes, including trnT, trnK, and ATP8. Comparison with other Microtus mitochondrial genomes ( $M$. ochrogaster; NC_027945.1 and M. fortis; NC_015243.1) showed that these insertions were only present in the Novoplasty mitochondrial assemblies. Therefore, the mapping assemblies were used for further analyses. The mapping assemblies for both species included ambiguous bases, which were much more frequent for M. montanus than M. $r$. arvicoloides. These could be the result of using the mitochondrial genome of a different subspecies (for M. r. arvicoloides) or species (for M. montanus) for mapping the reads. Additionally, the presence of nuclear DNA of mitochondrial origin (NUMTs [53, 54]) might have influenced these results. If mitochondrial segments have been incorporated into the nuclear genomes and subsequent mutations have occurred, both nuclear and mitochondrial sequences could be mapped to the same mitochondrial region during assembly, resulting in the ambiguous bases observed here. NUMTs are likely to be present, as they have been documented in other species of Microtus [55-57]. Both mitochondrial genomes were annotated using MITOS [58]. The annotations each consisted of 22 tRNA genes, two rRNA genes, and 13 protein-coding genes. 


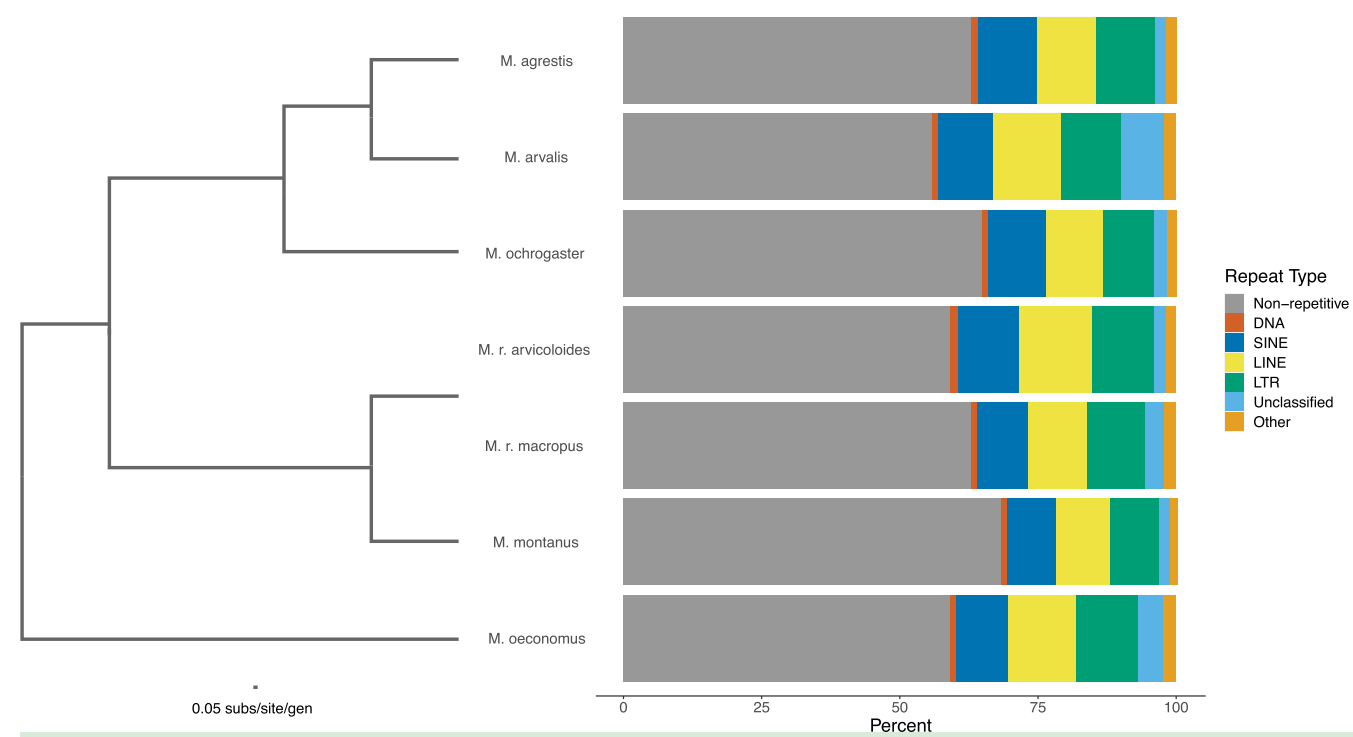

Figure 1. Repeat content among Microtus genomes. SINE: short interspersed nuclear element; LINE: long interspersed nuclear element; LTR: long terminal repeat; Other consists of small RNA, satellite, simple, and low complexity repeats. The phylogeny displayed was recreated from [3] by pruning unincluded species from the data alignment and rerunning RAxML [77] with the same settings used in the original analysis.

\section{Microtus genome assembly comparison}

The available Microtus genome assemblies, M. agrestis (GCA_902806755.1), M. arvalis (GCA_007455615.1), M. ochrogaster (GCA_000317375.1), and M. oeconomus

(GCA_007455595.1), were downloaded from GenBank. Assembly summary statistics were calculated using QUAST, bbmap, and custom Python scripts [46]. To compare repeat content among all genomes, including the three produced by the current study, repeats were first identified de novo using RepeatModeler v. 2.0.1 (RepeatModeler, RRID:SCR_015027) [59]. RepeatMasker v. 4.0.9 (RepeatMasker, RRID:SCR_012954) was then used to further identify repeats using a combined repeat library that included the repeats identified from RepeatModeler and those from the RepeatMasker Rodentia database [60]. The percentage of the genome comprising each type of repeat element was extracted from the RepeatMasker log file for each genome assembly.

All genome assemblies used some form of Illumina sequencing (Table 2), although assembly continuity varied greatly among assemblies, from 1366 scaffolds in M. agrestis to 1.6 million scaffolds in M. r. macropus. Genome coverage was similarly varied, from $13 \times$ in M. montanus, to $35 \times$ in M. r. macropus, to $77 \times$ in M. arvalis and M. oeconomus. The percentage of repetitive regions ranged from $31.7 \%$ in $M$. montanus to $44.1 \%$ in $M$. arvalis (Figure 1). Repeat content did not appear to be associated with phylogenetic relatedness, since repeats between the two subspecies of $M$. richardsoni were not more similar to each other than to other Microtus species. However, it is possible that the repeat content is affected by the continuity of the genome assemblies. Further research is needed to confirm this relationship.

\section{Genome annotation}

The M. $r$. arvicoloides genome assembly was annotated with the MAKER v. 2.31.10 (MAKER, RRID:SCR_005309) pipeline [61], loosely following the tutorial by Daren Card [62]. Briefly, 


\begin{tabular}{|c|c|c|c|c|c|c|c|}
\hline Species & M. agrestis & M. arvalis & M. montanus* & M. ochrogaster & M. oeconomus & $\begin{array}{l}\text { M. } r \text {. } \\
\text { arvicoloides* }\end{array}$ & M. r. macropus* \\
\hline Distribution & Europe & Europe & North America & North America & North America & North America & North America \\
\hline Year & 2020 & 2019 & 2020 & 2012 & 2019 & 2020 & 2020 \\
\hline BioProject & PRJEB36805 & PRJNA551185 & PRJNA673873 & PRJNA72443 & PRJNA551187 & PRJNA673719 & PRJNA509068 \\
\hline Sequencing & $\begin{array}{l}\text { 10× Chromium }+ \\
\text { Illumina }\end{array}$ & Illumina & Illumina & Illumina & Illumina & $\begin{array}{l}\text { 10 } \times \text { Chromium }+ \\
\text { Illumina }\end{array}$ & Illumina \\
\hline Assembler & Supernova & Discovar & RaGOO & ALLPATHS & Discovar & Supernova & Discovar \\
\hline Length (Gb) & 2.03 & 2.62 & 2.34 & 2.29 & 2.31 & 2.36 & 2.54 \\
\hline Coverage $(x)$ & 50 & 77 & 13 & 94 & 77 & 47 & 35 \\
\hline Scaffolds (n) & 1366 & $1,081,432$ & 12,962 & 6341 & 562,436 & 31,632 & $1,648,927$ \\
\hline $\begin{array}{l}\text { Longest scaffold } \\
(\mathrm{Mb})\end{array}$ & 56.96 & 0.80 & 748.72 & 126.73 & 0.93 & 16.00 & 0.26 \\
\hline N50 (Mb) & 13.35 & 0.53 & 3.08 & 61.81 & 0.11 & 2.30 & 0.02 \\
\hline L50 (n) & 45 & 11,870 & 91 & 14 & 5,556 & 278 & 35,660 \\
\hline N (\%) & 2.87 & 0.07 & 8.81 & 8 & 0.12 & 1.29 & 0.06 \\
\hline GC (\%) & 42.33 & 41.71 & 42.38 & 42.25 & 42.18 & 42.21 & 42.13 \\
\hline
\end{tabular}

the pipeline comprises masking repeats followed by multiple rounds of annotation, with both evidence-based and ab initio gene models. Repeats were identified as described above. Complex repeats were then extracted from RepeatMasker results using grep with keywords "Satellite" and "rich". Within Maker, the model_org argument was set to "simple" so Maker would soft-mask simple repeats, and the RepeatMasker results were provided to hard-mask complex repeats. Evidence-based gene discovery used protein and mRNA sequences from the previous genome annotation of M. ochrogaster (GCF_000317375.1), as well as an additional RNA-Seq assembly from M. pennsylvanicus (GSM3499528 [63]). Hidden Markov models (HMMs) for ab initio gene prediction were trained using both SNAP and Augustus v. 2.5.5 (Augustus: Gene Prediction, RRID:SCR_008417) [64, 65]. With SNAP, gene models identified by MAKER were filtered using an annotation edit distance (AED) of 0.5 and an amino acid length of 50. After validating these models with SNAP's Fathom utility, removing likely errors, and including $1000 \mathrm{bp}$ surrounding each training sequence, the training sequences were passed to the hmm-assembler script. For Augustus, training sequences plus $1000 \mathrm{bp}$ on each side were obtained from the first round of MAKER mRNA annotations. Augustus was used to train the HMM using the -long option in BUSCO and the Euarchontoglires reference set. MAKER was then run again with the previously annotated gene models, and the HMM models from SNAP and Augustus. After the initial MAKER run, two cycles of $a b$ initio gene prediction and annotation with MAKER were performed. To prevent overfitting, results were compared after each round of MAKER. Because the increase in AED score was minimal between the first and second rounds of $a b$ initio gene prediction, further analysis was conducted on the results after the first round only. This round annotated $\sim 24,500$ genes, with a mean gene length of $7445 \mathrm{bp}$ (Table 3 ), which is within the range found in previous studies of $M$. ochrogaster (22,427 genes;

GCF_000317375.1) and Arvicola amphibious (25,136 genes; GCF_903992535.1). Of these annotations, all occurred on scaffolds greater than $1 \mathrm{~Kb}$ in length, and $97 \%$ occurred on scaffolds greater than $10 \mathrm{~Kb}$ in length.

Functional annotation of the M. r. arvicoloides genome was performed using GOfeat [66], an online functional annotation tool that uses multiple protein databases including 


\begin{tabular}{cccc}
\hline \multirow{2}{*}{ Table 3. Structural annotation summary after each round of MAKER. } \\
\hline & Before gene modeling & Gene modeling round 1 & Gene modeling round 2 \\
\hline & 20,945 & 24,548 & 23,811 \\
Genes $(n)$ & 139,845 & 192,974 & 179,225 \\
Exons $(n)$ & 20,945 & 24,548 & 23,811 \\
mRNA $(n)$ & - & 24,504 & 24,539 \\
tRNA $(n)$ & - & 1229 & 1180 \\
\hline 5' UTR $(n)$ & - & 503 & 642 \\
\hline 3' UTR $(n)$ & - & 7445 & 7132 \\
Mean gene length (bp) & 0.993 & 0.881 & 0.888 \\
AED $<0.50$ (proportion) & 0.672 & 0.543 & 0.520 \\
\hline AED $<0.25$ (proportion) & - & 67.7 & 70.5 \\
\hline BUSCO (Complete) (\%) & & & \\
\hline UTR: untranslated region; AED: annotation edit distance. Values with dashes were not analyzed prior to gene \\
modeling with SNAP and Augustus.
\end{tabular}

UniProt [67], InterPro[68], and Pfam [69]. An input file for GOfeat was generated by supplying the genome assembly FASTA file and the MAKER General Feature Format (GFF3) file to the Python package gffread v. 0.12.1 [70]. GOfeat annotated $83.49 \%$ of genes. Biological processes accounted for $42.46 \%$ of annotations, cellular components accounted for $30.29 \%$, and molecular functions comprised $27.25 \%$. The most frequent gene ontology (GO) terms were 'positive regulation of transcription by RNA polymerase II', 'negative regulation of transcription by RNA polymerase II', and 'DNA-templated regulation of transcription' for biological processes; 'cytoplasm' and 'plasma membrane' for cellular components, and 'metal ion binding' and 'calcium ion binding' for molecular functions.

\section{DATA VALIDATION AND QUALITY CONTROL}

As described above, BUSCO was used to assess the quality of each genome assembly. Of the three final assemblies, two (M. r. arvicoloides and M. montanus) had BUSCO completeness scores over $80 \%$. Alternatively, the low BUSCO score for the best M. $r$. macropus assembly shows that while it can still be useful for mapping reads, the assembly could be substantially improved by collecting additional data. Additionally, a high percentage of reads mapped back to the $M$. montanus genome, suggesting that the assembly is largely complete despite using the M. r. arvicoloides assembly as a reference.

\section{REUSE POTENTIAL}

The current study details the assembly and annotation of three nuclear and two mitochondrial genomes. Compared with previously published nuclear genomes, the $M . r$. arvicoloides and $M$. montanus genomes are of high quality, as evidenced by the low number of scaffolds, high N50/L50 values, and high BUSCO scores. While not as complete as other Microtus genomes, the nuclear genome of M. r. macropus will still be useful for mapping low-coverage reads or reduced representation sequencing data. Furthermore, the mitochondrial genomes contributed here add to a growing number for the genus Microtus, and reinforce earlier suggestions that high-quality mitochondrial genomes can be obtained as byproducts of nuclear sequencing (e.g. [71, 72]). Overall, the data presented serve as an example that even though they do not include chromosomal information, high-quality draft genomes can be produced from widely available and very cost-effective methods, like the $10 \times$ Chromium protocol. These references can aid various studies, including those examining genus and species adaptation [73, 74], phylogenetics [21], phylogeography [24, 
75], and disease dynamics [6, 76]. However, some activities, like exploring changes to chromosome structure, will not be possible owing to fragmentation and the lack of chromosomal mapping for these assemblies. Finally, the M. r. macropus and M. montanus sequencing data and preliminary assemblies will serve as building blocks for more accurate reference genomes in the future.

\section{DATA AVAILABILITY}

Raw sequences, nuclear assemblies, and mitochondrial assemblies are available from GenBank under BioProjects PRJNA673719, PRJNA509068, and PRJNA673873 for M. $r$. arvicoloides, M. r. macropus, and M. montanus respectively. The custom python script used to calculate genome assembly summary information is available on GitHub [46]. Other supporting data, including assemblies, full BUSCO tables, structural annotation gff files, functional annotation tables, and repeat libraries are available in the GigaScience data repository GigaDB (M. richardsoni [78], M. montanus [79]).

\section{DECLARATIONS}

\section{LIST OF ABBREVIATIONS}

BOLD: Barcode of Life Database; bp: base pair; COI: cytochrome oxidase I; Gb: gigabase pairs; Mb: megabase pairs; Kb: kilobase pairs; AED: annotation edit distance; HMM: hidden Markov model; NUMT: nuclear DNA of mitochondrial origin.

\section{ETHICAL APPROVAL}

Not applicable.

\section{CONSENT FOR PUBLICATION}

Not applicable.

\section{COMPETING INTERESTS}

SP is the director of Iridian Genomes, Inc. The other authors declare that they have no competing interests.

\section{FUNDING}

Sequencing was funded by Iridian Genomes, Inc., as well as the National Science Foundation (DEB-1457519). Salary support for DD was provided by The Ohio State University and the National Science Foundation (DBI-1945347).

\section{AUTHORS' CONTRIBUTIONS}

DD, JS, and BC conceived the study. JS, SP, and BC provided funding for sequencing. DD performed DNA extractions, assembled genomes, and annotated genomes with input from SP. DD and BC wrote the manuscript with input from JS and SP. DD and SP submitted the resources to GenBank. All authors approved the final version.

\section{ACKNOWLEDGEMENTS}

We thank Jeffrey Good and Eric Rickart/Utah Museum of Natural History for tissue samples, Michael Broe for advice with genome assembly and annotation, and the Ohio Supercomputer Center (OSC) for computational resources. 


\section{REFERENCES}

1 Wilson DE, Reeder DM (eds) Mammal Species of the World: A Taxonomic and Geographic Reference, 3rd ed, vol. 2, Baltimore, MD: Johns Hopkins University 2005; pp. 956-1039.

2 Reig $\mathbf{O A}$, Karyotypic repatterning as one triggering factor in cases of explosive speciation. In: Fontdevila A (ed.), Evolutionary biology of transient unstable populations. Berlin, Heidelberg: Springer 1989.; pp. 246-289.

3 Steppan SJ, Schenk JJ, Muroid rodent phylogenetics: 900-species tree reveals increasing diversification rates. PLoS ONE, 2017; 12(8): e0183070.

4 Jackson DJ, Cook JA, A precarious future for distinctive peripheral populations of meadow voles (Microtus pennsylvanicus). J. Mammal., 2020; 101(1): 36-51.

5 Monarca RI, Speakman JR, da Luz Mathias M, Energetics and thermal adaptation in semifossorial pine-voles Microtus lusitanicus and Microtus duodecimcostatus. J. Comp. Physiol. B, 2019; 189(2): 309-318.

6 Wanelik KM et al. A candidate tolerance gene identified in a natural population of field voles (Microtus agrestis). Mol. Ecol., 2018; 27(4): 1044-1052.

7 Seelke AM, Perkeybile AM, Grunewald R, Bales KL, Krubitzer LA, Individual differences in cortical connections of somatosensory cortex are associated with parental rearing style in prairie voles (Microtus ochrogaster). J. Comp. Neurol., 2016; 524(3): 564-577.

8 Oli MK, Population cycles in voles and lemmings: state of the science and future directions. Mamm. Rev., 2019; 49(3): 226-239.

9 McGraw LA et al. Development of genomic resources for the prairie vole (Microtus ochrogaster): construction of a BAC library and vole-mouse comparative cytogenetic map. BMC Genom., 2010; 11(1): $1-8$.

10 Klaus M, Beauvais GP, Water Vole (Microtus richardsoni): a technical conservation assessment. USDA Forest Service, Rocky Mountain Region. (2004, January 20); http://www.fs.fed.us/r2/projects/scp/assessments/watervo Accessed 1st March 2021.

11 Stein BR, Bone density and adaptation in semiaquatic mammals. J. Mammal., 1989; 70(3): 467-476.

12 Dunstone N, Gorman ML (eds) Behaviour and Ecology of Riparian Mammals. Cambridge: Cambridge University Press 2007; ISBN:9780521038072.

13 Beichman AC et al. Aquatic adaptation and depleted diversity: a deep dive into the genomes of the sea otter and giant otter. Mol. Biol. E, 2019; 36(12): 2631-2655.

14 Ludwig DR, Microtus richardsoni. Mamm. Species, 1984; 223: 1-6.

15 Klaus M, Moore RE, Vyse E, Impact of precipitation and grazing on the water vole in the Beartooth Mountains of Montana and Wyoming, USA. Arct. Antarct. Alp. Res., 1999; 31(3): 278-282.

16 Cassola F, Microtus richardsoni. IUCN Red List of Threatened Species. 2016; doi:10.2305/IUCN.UK.2016-3.RLTS.T42634A22345832.en.

17 Wyoming Natural Diversity Database. University of Wyoming. 2020; https://wyndd.org/species_list/. Accessed 06 May 2020.

18 Sera WE, Early CN, Microtus montanus. Mamm. Species, 2003; 716: 1-10.

19 Cassola F, Microtus montanus. IUCN Red List of Threatened Species. 2016; doi:10.2305/IUCN.UK.2016-2.RLTS.T42630A22346732.en.

20 Bailey V, Revision of American voles of the genus Microtus. North American Fauna, 1900; 17: 1-88.

21 Barbosa S, Paupério J, Pavlova SV, Alves PC, Searle JB, The Microtus voles: Resolving the phylogeny of one of the most speciose mammalian genera using genomics. Mol. Phylogenet. Evol., 2018; 125: 85-92.

22 Cartens BC, Brunsfeld SJ, Demboski JR, Good JM, Sullivan J, Investigating the evolutionary history of the Pacific Northwest mesic forest ecosystem: hypothesis testing within a comparative phylogeographic framework. Evolution, 2005; 59(8): 1639-1652.

23 Carstens BC, Richards CL, Integrating coalescent and ecological niche modeling in comparative phylogeography. Evolution, 2007; 61(6): 1439-1454.

24 Espíndola A, Ruffley M, Smith ML, Carstens BC, Tank DC, Sullivan J, Identifying cryptic diversity with predictive phylogeography. Proc. Royal Soc. B, 2016; 283(1841): 20161529. 
25 Zheng GX et al. Haplotyping germline and cancer genomes with high-throughput linked-read sequencing. Nat. Biotechnol., 2016; 34(3): 303-311.

26 Weisenfeld NI, Kumar V, Shah P, Church DM, Jaffe DB, Direct determination of diploid genome sequences. Genome Res., 2017; 27(5): 757-767.

27 Luo R, Liu B, Xie Y, Li Z, Huang W, Yuan J, SOAPdenovo2: an empirically improved memory-efficient short-read de novo assembler. Gigascience, 2012; 1(1): 2047-2017.

28 Weisenfeld NI et al. Comprehensive variation discovery in single human genomes. Nat. Genet., 2014; 46(12): 1350.

29 Andrews S, FastQC: a quality control tool for high throughput sequence data. 2010; https://www.bioinformatics.babraham.ac.uk/projects/fastqc/.

30 Bolger AM, Lohse M, Usadel B, Trimmomatic: a flexible trimmer for Illumina sequence data. Bioinformatics, 2014; 30(15): 2114-2120.

31 Chikhi R, Medvedev P, Informed and automated k-mer size selection for genome assembly. Bioinformatics, 2014; 30(1): 31-37.

32 Yeo S, Coombe L, Warren RL, Chu J, Birol I, ARCS: scaffolding genome drafts with linked reads. Bioinformatics, 2018; 34(5): 725-731.

33 Warren RL et al. LINKS: scalable, alignment-free scaffolding of draft genomes with long reads. GigaScience, 2015; 4: 35.

34 Li H, Durbin R, Fast and accurate long-read alignment with Burrows-Wheeler transform. Bioinformatics, 2010; 26(5): 589-595.

35 Danecek P, Bonfield JK, Liddle J, Marshall J, Ohan V, Pollard MO, Whitwham A, Keane T, McCarthy SA, Davies RM, Li H, Twelve years of SAMtools and BCFtools. Gigascience, 2021; 10(2): giab008.

36 Ratnasingham S, Hebert PD, BOLD: The Barcode of Life Data System (http://www.barcodinglife.org). Mol. Ecol. Notes, 2007; 7(3): 355-364.

37 Alqahtani F, Duckett D, Pirro S, Mandoiu II, Complete mitochondrial genome of the water vole Microtus richardsoni (Cricetidae, Rodentia). Mitochondrial. DNA B Resour., 2020; 5(3): 2498-2499.

38 Broad Institute. Picard tools. 2016; http://github.com/broadinstitute/picard.

39 Li H, A statistical framework for SNP calling, mutation discovery, association mapping and population genetical parameter estimation from sequencing data. Bioinformatics, 2011; 27(21): 2987-2993.

40 Alonge $\mathrm{M}$ et al. RaGoO: fast and accurate reference-guided scaffolding of draft genomes. Genome Biol., 2019; 20(1): 1-7.

41 Modi WS, Phylogenetic analyses of chromosomal banding patterns among the Nearctic Arvicolidae (Mammalia: Rodentia). Syst. Biol., 1987; 36(2): 109-136.

42 Barnett DW, Garrison EK, Quinlan AR, Strömberg MP, Marth GT, BamTools: a C++ API and toolkit for analyzing and managing BAM files. Bioinformatics, 2011; 27(12): 1691-1692.

43 Benson DA et al. GenBank. Nucleic Acids Res., 2012; 41(D1): D36-D42.

44 Gurevich A, Saveliev V, Vyahhi N, Tesler G, QUAST: quality assessment tool for genome assemblies. Bioinformatics, 2013; 29(8): 1072-1075.

45 Bushnell B, BBMap: a fast, accurate, splice-aware aligner. Sourceforge. 2014; https://sourceforge.net/projects/bbmap/.

46 Duckett DJ, (n.d.). Genome resources. Github; https://github.com/djlduckett/Genome_Resources.

47 Simão FA, Waterhouse RM, Ioannidis P, Kriventseva EV, Zdobnov EM, BUSCO: assessing genome assembly and annotation completeness with single-copy orthologs. Bioinformatics, 2015; 31(19): 3210-3212.

48 Mikheenko A, Prjibelski A, Saveliev V, Antipov D, Gurevich A, Versatile genome assembly evaluation with QUAST-LG. Bioinformatics, 2018; 34(13): i142-i150.

49 Fernandez-Silva I, Henderson JB, Rocha LA, Simison WB, Whole-genome assembly of the coral reef Pearlscale Pygmy Angelfish (Centropyge vrolikii). Sci. Rep., 2018; 8: 1498.

50 Dierckxsens N, Mardulyn P, Smits G, NOVOPlasty: de novo assembly of organelle genomes from whole genome data. Nucleic Acids Res., 2017; 45(4): e18. 
51 Kearse $\mathbf{M}$ et al. Geneious Basic: an integrated and extendable desktop software platform for the organization and analysis of sequence data. Bioinformatics, 2012; 28(12): 1647-1649.

52 Edgar Rc, MUSCLE: multiple sequence alignment with high accuracy and high throughput. Nucleic Acids Res., 2004; 32(5): 1792-1797.

53 Lopez JV, Yuhki N, Masuda R, Modi W, O’Brien SJ, Numt, a recent transfer and tandem amplification of mitochondrial DNA to the nuclear genome of the domestic cat. J. Mol. Evol., 1994; 39(2): 174-190.

54 Hazkani-Covo E, Zeller RM, Martin W, Molecular poltergeists: mitochondrial DNA copies (nuMountains) in sequenced nuclear genomes. PLoS Genet., 2010; 6(2): e1000834.

55 Triant DA, DeWoody JA, Extensive mitochondrial DNA transfer in a rapidly evolving rodent has been mediated by independent insertion events and by duplications. Gene, 2007; 401(1-2): 61-70.

56 Triant DA, DeWoody JA, Molecular analyses of mitochondrial pseudogenes within the nuclear genome of arvicoline rodents. Genetica, 2008; 132(1): 21-33.

57 Triant DA, DeWoody JA, Demography and phylogenetic utility of numt pseudogenes in the Southern Red-Backed Vole (Myodes gapperi). J. Mammal., 2009; 90(3): 561-570.

58 Bernt M et al. MITOS: improved de novo metazoan mitochondrial genome annotation. $\mathrm{Mol}$. Phylogenet. E, 2013; 69(2): 313-319.

59 Smit AF, Hubley R, RepeatModeler Open-1.0. 2008; http://www.repeatmasker.org/RepeatMasker/.

60 Smit AF, Hubley R, Green P, RepeatMasker Open-4.0. 2013;

http://www.repeatmasker.org/RepeatModeler/.

61 Cantarel BL et al. MAKER: an easy-to-use annotation pipeline designed for emerging model organism genomes. Genome Res., 2008; 18(1): 188-196.

62 Card D., (n.d.) Genome Annotation using MAKER;

https://gist.github.com/darencard/bb1001ac1532dd4225b030cf0cd61ce2.

63 Young RL et al. Conserved transcriptomic profiles underpin monogamy across vertebrates. Proc. Natl Acad. Sci. USA, 2019; 116(4): 1331-1336.

64 Korf I, Gene finding in novel genomes. BMC Bioinform., 2004; 5(1): 59.

65 Stanke M, Waack S, Gene prediction with a hidden Markov model and a new intron submodel. Bioinformatics, 2003; 19(ii): 215-225.

66 Araujo FA, Barh D, Silva A, Guimarães L, Ramos RT, GO FEAT: a rapid web-based functional annotation tool for genomic and transcriptomic data. Sci. Rep., 2018; 8(1): 1-4.

67 UniProt Consortium. UniProt: a hub for protein information. Nucleic Acids Res., 2015; 43(D1): D204-D212.

68 Hunter S et al. InterPro: the integrative protein signature database. Nucleic Acids Res., 2009; 37: D211-D215.

69 Bateman A et al. The Pfam protein families database. Nucleic Acids Res., 2004; 32: D138-D141.

70 Pertea G, Pertea M, GFF Utilities: GffRead and GffCompare. F1000Research. 2020; 9.

71 Voigt O, Erpenbeck D, Wörheide G, A fragmented metazoan organellar genome: the two mitochondrial chromosomes of Hydra magnipapillata. BMC Genom., 2008; 9(1): 350.

72 Smith DR, Not seeing the genomes for the DNA. Brief. Funct. Genom., 2012; 11(4): 289-290.

73 Fink S, Excoffier L, Heckel G, Mitochondrial gene diversity in the common vole Microtus arvalis shaped by historical divergence and local adaptations. Mol. Ecol., 2004; 13(11): 3501-3514.

74 Fischer MC, Foll M, Excoffier L, Heckel G, Enhanced AFLP genome scans detect local adaptation in high-altitude populations of a small rodent (Microtus arvalis). Mol. Ecol., 2011; 20(7): 1450-1462.

75 Frey JK, Genetics of allopatric populations of the montane vole (Microtus montanus) and Mogollon vole (Microtus mogollonensis) in the American Southwest. West. N. Am. Nat., 2009; 69(2): 215-222.

76 Tołkacz K et al. Bartonella infections in three species of Microtus: prevalence and genetic diversity, vertical transmission and the effect of concurrent Babesia microti infection on its success. Parasites Vectors, 2018; 11: 491 
77 Silvestro D, Michalak I, raxmlGUI: a graphical front-end for RAxML. Org. Divers. Evol., 2012; 12(4): 335-337.

78 Duckett DJ, Sullivan J, Pirro S, Carstens BC, Genomic data for the North American water vole (Microtus richardsoni). GigaScience Database. 2021; http://dx.doi.org/10.5524/100886.

79 Duckett DJ, Sullivan J, Pirro S, Carstens BC, Genomic data for the montane vole (Microtus montanus). GigaScience Database. 2021; http://dx.doi.org/10.5524/100885. 